\title{
ESTILO DE VIDA E LONGEVIDADE NA QUALIDADE DA SAÚDE DA PESSOA IDOSA
}

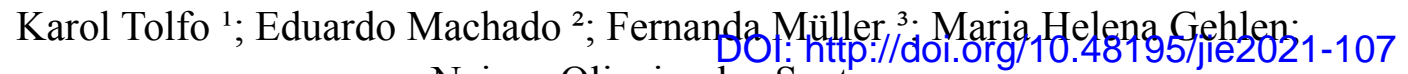 \\ Naiana Oliveira dos Santos
}

karoltolfo@hotmail.com ${ }^{1}$; eduardomaruqes051@.gmail.com ${ }^{2}$; fcopettimuller@gmail.com ${ }^{3}$ gehlenmh@gmail.com ${ }^{4}$; naiana.santos@ufn.edu.br ${ }^{5}$

\section{RESUMO}

O envelhecimento é um processo gradual, universal e irreversível, provocando uma perda funcional progressiva no organismo, oriunda de alterações orgânicas, como a redução do equilíbrio e mobilidade, das capacidades fisiológicas e psicológicas (GEIB, 2012; MACIEL, 2010). Objetiva-se descrever a importância do estilo de vida na promoção da longevidade; Trata-se de uma revisão narrativa realizado durante uma atividade de ensino, para a disciplina de Atenção Integral à Saúde do Idoso, referente a blue zones. O estilo de vida tem suma importância no processo de envelhecimento ao estimular a longevidade com funcionalidade. Conclui-se que as condições e estilo de vida adotados ao longo da vida refletem no processo de envelhecimento, assim se deve, a importância da promoção da saúde juntamente com seu estilo de vida. Por fim, o acompanhamento multiprofissional se torna indispensável no processo de envelhecimento de forma saudável.

Descritores: Geriatria; Longevidade; Qualidade de Vida.

\section{INTRODUÇÃO}

A saúde é influenciada por diferentes agentes ao longo da vida, vinculados ao contexto social, que geram desigualdades nas exposições e vulnerabilidades. Os determinantes sociais têm um papel importante quando se trata de estilo de vida e longevidade, visto que, interferem no bem-estar, na independência social e na qualidade de vida da pessoa idosa. O envelhecimento é um processo gradual, universal e irreversível, provocando uma perda funcional progressiva no organismo, oriunda de alterações orgânicas, 
como a redução do equilíbrio e mobilidade, das capacidades fisiológicas e psicológicas (GEIB, 2012; MACIEL, 2010).

A longevidade e o quantitativo de pessoas idosas é crescente, visto que, é um fenômeno populacional irreversível em diversas mudanças na sociedade, como: a diminuição das taxas de fecundidade, a queda da mortalidade, o aumento da expectativa de vida e o melhor acesso às tecnologias e aos serviços de saúde. Outrossim, nesse sentido é possível observar a necessidade e a importância da adequação da sociedade a fim de promover e garantir um envelhecimento saudável e ativo, buscando assim a qualidade de vida (LIMA, et al., 2017).

Envelhecer com qualidade de vida deve ser uma prioridade em saúde pública, para isso existem fatores a serem observados como às condições sociais, diversidade cultural e regiões de moradia. A população idosa brasileira residente na zona urbana que não está a beira da vulnerabilidade dispõe de melhor acesso aos serviços de saúde, esses fatores devem ser considerados em políticas públicas e nas diferenças de qualidade de vida (SAMPAIO, et al., 2017).

A busca pelo envelhecimento longevo com qualidade de vida se torna um amplo anseio de toda a sociedade. Para isso tem se utilizado a abordagem multidimensional para o cuidado com a pessoa idosa. Para garantir longevidade com qualidade de vida também se torna necessário proporcionar condições para passar a viver a longevidade com qualidade de vida, associando a autonomia, bem-estar, suporte social e acessibilidade (PEREIRA, NOGUEIRA, SILVA, 2015).

A enfermagem gerontológica, além de ser a ciência do cuidado, acompanha o processo de envelhecimento com o cuidado em todos os níveis. Assim, assume um caráter multidisciplinar e multidimensional, percebendo qual a limitação do idoso e de forma individual, buscando proporcionar qualidade de vida com autonomia e funcionalidade (DA VEIGA et al., 2021).

Frente ao exposto, o presente trabalho tem como objetivo descrever a importância do estilo de vida na promoção da longevidade.

\section{METODOLOGIA}

Trata-se de um estudo de revisão narrativa de literatura, realizado durante uma atividade de ensino, para a disciplina de Atenção Integral à Saúde do Idoso, referente a blue zones. O estudo foi desenvolvido entre os meses de maio e junho do ano de 2020. De tal 
modo, por meio remoto se desenvolveu o trabalho descritivo e após um card para visibilidade do estilo de vida na promoção da longevidade, sendo este socializado nas redes sociais.

Além disso, foi realizada a busca de artigos acadêmicos nas plataformas, Biblioteca de Dados de Enfermagem (BDENF) e Lilacs, selecionando os materiais relativos a estilo de vida na promoção da longevidade. Quanto aos critérios de inclusão considerou-se: os artigos completos disponíveis em português que abordassem a temática com recorte temporal de dez anos, pelo fato de obter resultados atualizados. Os critérios de exclusão foram artigos que não estivessem disponíveis na íntegra, e os que não atendessem o objetivo proposto no trabalho. Com isso foram encontrados 14 trabalhos nas bases de dados utilizadas e selecionados sete trabalhos que compõem os resultados e discussões a seguir.

\section{RESULTADOS E DISCUSSÕES}

O estilo de vida tem suma importância no processo da longevidade. As ações cotidianas do estilo de vida dos idosos modificam suas relações de saúde, refletindo diretamente no processo de envelhecimento. Sejam ela fragilizando ou potencializando sua senescência (CASTRO, et. al, 2020). Essas ações, podem ser definitivas quanto ao envelhecimento, sendo por exemplo com o aparecimento de doenças crônicas ou com a manutenção da vitalidade.

Conforme a revisão integrativa de Melo, et al. (2019), a multimorbidade em pessoas com mais de 60 anos é ligada diretamente às questões socioeconômicas e hábitos como: tabagismo, consumo de álcool, residir em zonas rurais, baixa escolaridade, uso prévio e recente de serviços de saúde, gênero feminino, idosos mais velhos, estrutura familiar, polifarmácia e visão negativa da própria saúde.

Segundo estudo de Maia, et al. (2020), com pessoas entre 60 a 107 anos de idade no Brasil, para que haja um envelhecimento com sucesso é vinculado às capacidades físicas e mentais, o que se torna essencial para a autonomia e independência. Remetendo a um ambiente enriquecedor das dimensões humanas, o que possibilitam à essa população manter suas relações sociais e psíquicas.

Os pesquisadores ainda evidenciaram que aqueles participantes que possuíam como hábito de vida a dança eram mais otimistas e com mais perspectiva de vida, além de manterem uma robustez.

Observa-se que o sedentarismo é um fator predisponente chave para agravos no processo de envelhecimento, associando-se com o grau socioeconômico e local de moradia em que estes residem. Autores concluíram que os que possuíam maior renda, escolaridade e 
eram urbanizados, passavam maior parte de suas vidas assistindo televisão, contribuindo diretamente para a perda muscular e multimorbidades. Já aqueles moradores da zona rural, com menor escolaridade e renda, se mantiveram praticando as funcionalidades físicas ao decorrer dos anos, diminuindo os riscos decorrentes (LEÃO, KNUTH, MEUCCI, 2020).

Percebe-se que com o decorrer dos tempos há um grande aumento de tentativas de suicídio, suicídios e depressão em pessoas com mais de 60 anos. Vinculando-se ao enfrentamento do processo envelhecer e morrer, estrutura familiar, reflexões acerca do passado, questões econômicas e fragilidade funcional, psíquica e social. Essas questões também podem ter relação com o isolamento social, visto que com a transição demográfica e urbanização, esse isolamento se torna uma problemática.

Para Minayo, Figueiredo e Mangas (2019), a ideação suicida e a negligência são muito frequentes dentro das Instituições de Longa Permanência para Idosos - ILPIs. Assim, é preciso organizar um trabalho de prevenção efetivo para essa população, desde atividades e interações em grupos, para incentivar as relações sociais, psicoterapia, terapias medicamentosas para alguns casos.

Em uma revisão integrativa, realizada em 2017, foi possível identificar a religiosidade e espiritualidade, como fatores que proporcionam estilos de vida mais saudáveis. Onde esses fatores ajudam em situações estressantes e contribuem para situações de adversidades (TAVARES et al., 2017).

No estudo de Da Veiga, et al. (2021), que utiliza o Modelo Teórico de Nola Pender, entende que a promoção da saúde se trata da motivação de aumentar o bem-estar e valorizar o potencial para o estado de ótima saúde. Dessa forma, para o envelhecimento saudável, a família se torna parte primordial no processo de promoção à saúde, auxiliando um envelhecimento ativo e prazeroso.

Dessa forma, ressalta-se a extrema importância de analisar e construir planos de cuidados individualizados para essa população. Diferenciando suas singularidades e observando suas necessidades com o intuito de prover um processo de envelhecimento saudável.

\section{CONSIDERAÇÕES FINAIS}

Ao se descrever a importância do estilo de vida na promoção da longevidade, foi possível elencar que a fragilidade emocional do idoso poderá se acentuar, visto que o 
isolamento social, comorbidades, abandonos, reflexões sobre o passado, enfrentamento do processo de envelhecimento e da morte são pontos importantes nessa população.

Acrescenta-se que as condições e estilo de vida adotados ao longo da vida refletem diretamente o processo de envelhecimento. Sendo, dessa forma, indispensável o acompanhamento multiprofissional para um envelhecimento onde há prevenção, promoção e recuperação da saúde da pessoa idosa. Respeitando sua multidimensionalidade, autonomia, individualidade e direitos fundamentais.

\section{REFERÊNCIAS}

CASTRO, A. P. S., et. al. Vivendo en comunidad, envejeciendo de forma saludable. Enfermería Global, vol. 19, no.57 Murcia ene. 2020. Epub 16-mar-2020. Disponível em: $<\underline{\text { http://scielo.isciii.es/scielo.php?script }=\text { sci arttext\&pid }=S 1695-61412020000100010 \& l a n g=}$ $\underline{\text { en }}>$

DA VEIGA, D. O. C. et al. A promoção de saúde e seus impactos no envelhecimento ativo sob a ótica da teoria de Nola j. Pender: um relato histórico. Brazilian Journal of Health Review, vol.4, n.1, p.3240-3257, Curitiba, 2021.

GEIB, L. T. C. Determinantes sociais da saúde do idoso. Ciênc. saúde coletiva, Rio de Janeiro, v. 17, n. 1, p. 123-133, Jan. 2012.

LEÃO, O. A. A., KNUTH, A. G., MEUCCI, R. D. Comportamento sedentário em idosos residentes de zona rural no extremo sul do Brasil. Revista Brasileira de Epidemiologia, vol. 23, Rio de janeiro,2020, Epub Feb. 2, 2020. Disponível em: $<$ https://www.scielo.br/scielo.php?script=sci arttext\&pid=S1415-790X2020000100402\&lang $=\mathrm{em}>$

LIMA, W. R., et al. Características socioeconômicas, demográficas, de saúde e de longevidade de idosos: contribuição da enfermagem. International Nursing Congress. 2017

MACIEL, M. G. Atividade física e funcionalidade do idoso. Motriz: rev. educ. fis. (Online), Rio Claro, v. 16, n. 4, p. 1024-1032, Dec. 2010.

MAIA, L. C., et al. Robust older adults in primary care: factors associated with successful aging. Rev. Saúde Pública, 54, 06, Apr. 2020. Disponível em: $<\underline{\text { https://www.scielosp.org/article/rsp/2020.v54/35/> }}$

MINAYO, M. C. S., FIGUEIREDO, A. E. B., MANGAS, R. M. N. Estudo das publicações científicas (2002-2017) sobre ideação suicida, tentativas de suicídio e autonegligência de idosos internados em Instituições de Longa Permanência. Ciência \& Saúde Coletiva, vol. 24 no. 4, Rio de Janeiro Apr. 2019, Epub May 02, 2019. Disponível em: 
$<$ https://www.scielo.br/scielo.php?script=sci_arttext\&pid=S1413-81232019000401393\&lang

$=\mathrm{en}>$

MELO, L. A., et al. Fatores associados à multimorbidade em idosos: uma revisão integrativa da literatura. Revista Brasileira de Geriatria e Gerontologia. vol. 22, no.1, Rio de Janeiro, 2019, Epub June 10. Disponível em:

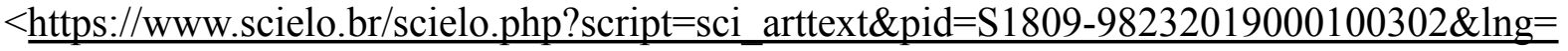
en\&nrm=iso\&tlng $=p t>$

PEREIRA, D. S.; NOGUEIRA, J. A. D.; SILVA, C. A. B. Qualidade de vida e situação de saúde de idosos: um estudo de base populacional no Sertão Central do Ceará. Rev. Bras. Geriatr. Gerontol., vol. 18, n. 4, p.893-908, Rio de Janeiro, 2015. da Conquista, 2017.

SAMPAIO, L. C. et al. Qualidade De Vida Em Idosos Residentes Em Zona Urbana E Rural. C\&D-Revista Eletrônica da FAINOR, v. 10, n. 3, p. 391-406, Vitória.

TAVARES, R. E. et al. Envelhecimento saudável na perspectiva de idosos: uma revisão integrativa. Rev. Bras. Geriatr. Gerontol., vol. 20, n. 6, p. 889-900, Rio de Janeiro, 2017 